\title{
SUPERSYMMETRIC SIGMA MODELS AND HARMONIC SUPERFUNCTIONS
}

\author{
JAIME MUÑOZ-MASQUÉ \\ Instituto de Física Aplicada \\ Consejo Superior de Investigaciones Científicas \\ Serrano 141 CP28006 Madrid (Spain) \\ jaime@iec.csic.es \\ JOSÉ ANTONIO VALLEJO \\ Facultad de Ciencias \\ Universidad Autónoma de San Luis Potosí \\ Salvador Nava s/n CP78290 San Luis Potosí (México) \\ jvallejo@fciencias.uaslp.mx
}

\begin{abstract}
We study the relation between the Laplacian associated to an odd metric on a supermanifold and harmonic superfunctions, through the application of the calculus of variations to a supersymmetric sigma model.
\end{abstract}

Keywords: Berezinian sheaf, harmonic superfunctions, supermanifolds, supersigma models.

\section{Introduction}

In the last years (since the apparition of the Batalin-Vilkovisky quantization method) there has been a certain amount of interest on Laplace operators of odd parity on supermanifolds, mainly in connection with their use in the quantization of non Abelian gauge theories (BRST symmetries, see [19]16/7/2].) These operators have been related to odd Poisson structures and odd divergences, modular classes, etc. (see $20[9] 8$.) But we want to focus here on the "Riemannian geometric" side of the problem, and the relation between Laplace's equation and harmonic functions (for a physical interpretation and relation to string theory, see [14.) In the classical setting, harmonic functions are obtained as follows. Consider two fixed Riemannian manifolds $(M, g)$ and $(N, h)$. Given a mapping $\phi:(M, g) \rightarrow(N, h)$, we can take the pullback of the metric $h$ by $\phi$ and then raise an index with the inverse metric $g^{-1}$. If $A=g^{-1} \cdot \phi^{*} h$ is the resulting bundle endomorphism $A: T M \rightarrow T M$, its trace $\operatorname{tr} A$ is a function on $M$ which can be integrated (with respect to the Riemannian volume of $g$ ) to give the functional

$$
\phi \mapsto \int_{M}(\operatorname{tr} A) \operatorname{vol}_{g}
$$


Critical points of this functional are called harmonic mappings (see [5]415].) In the particular case $N=\mathbb{R}$ they are also called harmonic functions, and the EulerLagrange equations for the above functional are precisely

$$
\triangle \phi=0,
$$

so that harmonic functions are the solutions to Laplace equations (where the Laplacian is understood as div o grad.) In Physics, the construction we have described is known as a sigma model, and harmonicity plays a very important rôle in their study. In this paper, we want to show that the relation between harmonicity and the Laplacian is preserved when passing to the supermanifold setting, and this is done by applying the methods of the calculus of variations to the sections of a graded submersion representing supersigma models.

We would like to stress two features of our approach:

(1) We use odd metrics.

(2) The superlaplacian $\triangle$ is not trivial, due to the fact that we use the Berezinian sheaf to define it (compare this with the geometric construction in [10, where any superfunction is harmonic).

To make the paper relatively self-contained, we include two brief sections on the Berezinian module and graded metrics on supermanifolds. Then we write our Lagrangian for the supersymmetric sigma model and associate to it a variational problem, so we can apply the techniques of the graded calculus of variations (for a detailed account of these techniques, and general definitions, notations and results on supermanifold theory, see [12]13] and references therein).

\section{2. $\mathbb{Z}_{2}$-graded metrics}

Let $(M, \mathcal{A})=(M, \wedge \mathcal{E})$ be an $(m \mid n)$-dimensional supermanifold given in Batchelor's form, so $\mathcal{E}=\Gamma(E)$ where $E \rightarrow M$ is a vector bundle. We denote by $\left\{\frac{\partial}{\partial x^{k}}\right\}_{k=1}^{m}$ a local frame on $M$, and by $\left\{x^{-j}\right\}_{j=1}^{n}$ a local frame of sections of $\mathcal{E}$. By $\sim$ we will understand the structural morphism of the supermanifold, $\sim: \mathcal{A} \rightarrow C^{\infty}(M)$.

As in [11], a $\mathbb{Z}_{2}$-graded metric (or supermetric) on $\wedge \mathcal{E}$ is understood to be a graded symmetric, non degenerate $\wedge \mathcal{E}$-bilinear map $G$ : Der $\wedge \mathcal{E} \times \operatorname{Der} \wedge \mathcal{E} \rightarrow \wedge \mathcal{E}$, whose action on a pair $\left(D_{1}, D_{2}\right) \in \operatorname{Der} \wedge \mathcal{E} \times \operatorname{Der} \wedge \mathcal{E}$ is denoted $\left\langle D_{1}, D_{2} ; G\right\rangle$ (for another approach to supermetrics, based on principal superbundles, see [18.) We have

(1) $\left\langle\alpha D_{1}, D_{2} ; G\right\rangle=\alpha\left\langle D_{1}, D_{2} ; G\right\rangle, \forall \alpha \in \wedge \mathcal{E}$.

(2) $\left\langle D_{1}, D_{2} ; G\right\rangle=(-1)^{\left|D_{1}\right|\left|D_{2}\right|}\left\langle D_{2}, D_{1} ; G\right\rangle$.

(3) The map $G^{b}: D \mapsto\langle D, . ; G\rangle$ is an isomorphism between the $\wedge \mathcal{E}$-modules $\operatorname{Der} \wedge \mathcal{E}$ and $\operatorname{Hom}(\operatorname{Der} \wedge \mathcal{E}, \wedge \mathcal{E})=\Omega_{G}^{1}(M)$. The inverse of this isomorphism is denoted by $G^{\sharp}$, as in the non-graded setting. Also, the induced metric on $\Omega_{G}^{1}(M)$ is denoted by $G^{-1}$. 
Note that these conditions imply $\left\langle D_{1}, \alpha D_{2} ; G\right\rangle=(-1)^{|\alpha|\left|D_{1}\right|}\left\langle D_{1}, D_{2} ; G\right\rangle$. Also, we remark that graded forms have a right $\wedge \mathcal{E}$-module structure, so we will take care in writting the $\wedge \mathcal{E}$ factors to the left of graded vector fields and to the right of graded forms.

A graded metric is even (resp. odd) if, $\left|\left\langle D_{1}, D_{2} ; G\right\rangle\right|+\left|D_{1}\right|+\left|D_{2}\right| \equiv 0 \bmod 2$ (resp. $\equiv 1 \bmod 2)$. In both cases the graded metric is called homogeneous. The associated concept of a graded connection is defined analogously to the non-graded case.

We focus our attention to graded metrics of second-order depth. This means that there is a graded basis (a set of generators of the locally finite $\wedge \mathcal{E}$-module $\operatorname{Der} \wedge \mathcal{E})\left\{D_{\alpha}\right\}_{\alpha=1}^{m+n}$ such that,

$$
\left\langle D_{i}, D_{j} ; G\right\rangle \in \sum_{0 \leq k \leq 2} \wedge^{k} \mathcal{E}
$$

In [11, Proposition 4.1] the following is proved:

Let $\langle\cdot, \cdot\rangle$ be a homogeneous graded metric of second order depth which is adapted to the canonical splitting of $\wedge \mathcal{E}$. A connection $\nabla$ exists on $M$ such that,

$$
\langle\cdot, \cdot ; G\rangle= \begin{cases}\left(\begin{array}{cc}
g & 0 \\
0 & \omega
\end{array}\right) & \text { with } \nabla g=0(\text { even case }) \\
\left(\begin{array}{cc}
0 & \kappa \\
\kappa^{t} & 0
\end{array}\right) & (\text { odd case },)\end{cases}
$$

with respect to the basis $\left\{\nabla_{\frac{\partial}{\partial x^{k}}}, i_{\frac{\partial}{\partial x^{-j}}}\right\}_{1 \leq k \leq m}^{1 \leq j \leq n}$ of Der $\wedge \mathcal{E}$, where $g$ is a metric on $T M, \omega$ is a symplectic form on $M$, and $\kappa: \overline{\mathcal{X}}(M) \rightarrow \mathcal{E}^{*}$ is a non-degenerate linear map.

If $d^{G}$ denotes the graded exterior derivative acting on the algebra of graded forms $\Omega_{G}(M)$-in order to distinguish it from the usual exterior derivative $d$, which acts on $\Omega(M)$ - and $\left\{d^{G} x^{k}, d^{G} x^{-j}\right\}_{1 \leq k \leq m}^{1 \leq j \leq n}$ is the dual basis on $\Omega_{G}^{1}(M)$ to the basis of derivations $\left\{\nabla_{\frac{\partial}{\partial x^{k}}}, i \frac{\partial}{\partial x^{-j}}\right\}_{1 \leq k \leq m}^{1 \leq j \leq n}$, we can write

$$
\langle., . ; G\rangle=d^{G} x^{i} \otimes d^{G} x^{-j} \cdot \kappa_{i j}+d^{G} x^{-i} \otimes d^{G} x^{j} \cdot \kappa_{j i} .
$$

for any odd metric on $(M, \wedge \mathcal{E})$. Note that, in the case $\mathcal{E}=\Gamma(T M)$, we can make the identifications $\nabla_{\frac{\partial}{\partial x^{k}}} \equiv \frac{\partial}{\partial x^{k}}$ and $i \frac{\partial}{\partial x^{-j}}=\frac{\partial}{\partial x^{-j}}$ as differential operators on the $C^{\infty}(M)$-algebra $\wedge \mathcal{E}$.

Example 1. Consider the linear supermanifold $\mathbb{R}^{1 \mid 1}=(\mathbb{R}, \Omega(\mathbb{R}))$ with global supercoordinates $\{t, \tau\},|t|=0,|\tau|=1$. The canonical odd Euclidean supermetric can be written as

$$
Q=d^{G} t \otimes d^{G} \tau+d^{G} \tau \otimes d^{G} t .
$$

Note that $\mathbb{R}^{1 \mid 1}$ admits no even supermetrics (this would require the base manifold being even dimensional). 
Example 2. Let $\mathcal{E}=\Gamma(T M)$ and let $g$ be a metric on $M$. A second-order depth odd supermetric $G$ on $(M, \wedge \mathcal{E})$ can naturally be defined by simply taking as $\kappa$ the induced isomorphism $g: \mathcal{X}(M) \rightarrow \Omega^{1}(M)$. In local coordinates:

$$
G=d^{G} x^{i} \otimes d^{G} x^{-j} \cdot g_{i j}+d^{G} x^{-i} \otimes d^{G} x^{j} \cdot g_{j i},
$$

where $g_{i j}$ is the matrix of $g$ with respect to the local frame $\left\{\frac{\partial}{\partial x^{k}}\right\}_{k=1}^{m}$ on $M$.

\section{The Berezinian sheaf and divergence}

Let $(M, \mathcal{A})$ be a graded manifold, of dimension $(m \mid n)$, and let $\mathrm{P}^{k}(\mathcal{A})$ be the sheaf of graded $k$-order differential operators of $\mathcal{A}$. This is the submodule of $\operatorname{End}(\mathcal{A})$ whose elements $P$ verify

$$
\left[\ldots\left[\left[P, a_{0}\right], a_{1}\right], \ldots, a_{k}\right]=0,
$$

for all $a_{0}, \ldots, a_{k} \in \mathcal{A}$ (here we identify an $a \in \mathcal{A}$ with the endomorphism $b \mapsto a b$ ).

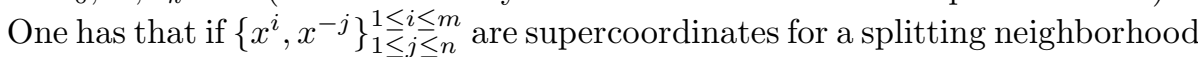
$U \subset M, \mathrm{P}^{k}(\mathcal{A}(U))$ is a free module (for both structures, left and right) with basis

$$
\frac{\partial^{|\alpha|}}{\partial x^{\alpha}} \circ \frac{\partial^{|\beta|}}{\partial x^{-\beta}}=\left(\frac{\partial}{\partial x^{1}}\right)^{\alpha_{1}} \circ \cdots \circ\left(\frac{\partial}{\partial x^{m}}\right)^{\alpha_{m}} \circ\left(\frac{\partial}{\partial x^{-1}}\right)^{\beta_{1}} \circ \cdots \circ\left(\frac{\partial}{\partial x^{-n}}\right)^{\beta_{n}},
$$

where $|\alpha|+|\beta| \leq k$.

Let us consider the sheaf $\mathrm{P}^{k}\left(\mathcal{A}, \Omega_{G}^{m}\right)=\Omega_{G}^{m} \otimes_{\mathcal{A}} \mathrm{P}^{k}(\mathcal{A})$, of $m$-form valued $k$-th order differential operators of $\mathcal{A}$. For every open subset $U \subset M$, let $\mathrm{K}^{n}(U)$ be the set of operators $P \in \mathrm{P}^{n}\left(\mathcal{A}(U), \Omega_{G}^{m}(U)\right)$ such that for every $a \in \mathcal{A}(U)$ with compact support, there exists an ordinary $(m-1)$-form of compact support, $\omega$, fulfilling $\widetilde{P(a)}=d \omega$. We observe that $\mathrm{K}^{n}$ is a submodule of $\mathrm{P}^{n}\left(\mathcal{A}, \Omega_{G}^{m}\right)$ for its right structure, so we can take quotients and obtain the following description of the Berezinian sheaf:

$$
\operatorname{Ber}(\mathcal{A})=\mathrm{P}^{n}\left(\mathcal{A}, \Omega_{G}^{m}\right) / \mathrm{K}^{n} .
$$

According to this description, a local basis of $\operatorname{Ber}(\mathcal{A})$ can be given explicitly: If $\left\{x^{i}, x^{-j}\right\}_{\substack{1 \leq i \leq m \\ 1 \leq j \leq n}}$ are supercoordinates for a splitting neighborhood $U \subset M$, the local sections of the Berezinian sheaf are written in the form

$$
\Gamma_{U}(\operatorname{Ber}(\mathcal{A}))=\left[d^{G} x^{1} \wedge \cdots \wedge d^{G} x^{m} \otimes \frac{\partial}{\partial x^{-1}} \circ \cdots \circ \frac{\partial}{\partial x^{-n}}\right] \cdot \mathcal{A}(U),
$$

where [ ] stands for the equivalence class modulo $\mathrm{K}^{n}$.

Now, if $X$ is a graded vector field, it is possible to define the notion of graded Lie derivative of sections of the Berezinian sheaf with respect to $X$. This is the mapping

$$
\mathcal{L}_{X}^{G}: \Gamma(\operatorname{Ber}(\mathcal{A})) \longrightarrow \Gamma(\operatorname{Ber}(\mathcal{A}))
$$

given by

$$
\mathcal{L}_{X}^{G}\left[\eta^{G} \otimes P\right]=(-1)^{|X|\left|\eta^{G} \otimes P\right|+1}\left[\eta^{G} \otimes P \circ X\right],
$$


for $X$ and $\eta^{G} \otimes P$ homogeneous.

This Lie derivative, has the properties that one would expect (cfr. the treatment in [3, Vol. 1 pg. 83):

(1) For homogeneous $X \in \operatorname{Der}(\mathcal{A}), \xi \in \Gamma(\operatorname{Ber}(\mathcal{A}))$ and $a \in \mathcal{A}$,

$$
\mathcal{L}_{X}^{G}(\xi \cdot a)=\mathcal{L}_{X}^{G}(\xi) \cdot a+(-1)^{|X||\xi|} \xi \cdot X(a) .
$$

(2) For homogeneous $X \in \operatorname{Der}(\mathcal{A}), \xi \in \Gamma(\operatorname{Ber}(\mathcal{A}))$ and $a \in \mathcal{A}$,

$$
\mathcal{L}_{a \cdot X}^{G}(\xi)=(-1)^{|X||\xi|} \mathcal{L}_{X}^{G}(\xi \cdot a) .
$$

(3) If $\xi_{x^{i}, x^{-j}}=\left[d^{G} x^{1} \wedge \cdots \wedge d^{G} x^{m} \otimes \frac{\partial}{\partial x^{-1}} \circ \cdots \circ \frac{\partial}{\partial x^{-n}}\right]$ is the local generator of the Berezinian sheaf on a system of supercoordinates $\left\{x^{i}, x^{-j}\right\}_{1 \leq j \leq n}^{1 \leq i \leq m}$, then

$$
\mathcal{L}_{\frac{\partial}{\partial x^{i}}}^{G}\left(\xi_{x^{i}, x^{-j}}\right)=0=\mathcal{L}_{\frac{\partial}{\partial x^{-j}}}^{G}\left(\xi_{x^{i}, x^{-j}}\right) .
$$

We can now introduce the notion of Berezinian divergence: Let $(M, \mathcal{A})$ be a graded manifold whose Berezinian sheaf is generated by a section $\xi$. The graded function $\operatorname{div}_{B}^{\xi}(X)$ given by the formula (for homogeneous $X$ )

$$
\mathcal{L}_{X}^{G}(\xi)=(-1)^{|X||\xi|} \xi \cdot \operatorname{div}_{B}^{\xi}(X)
$$

is called the Berezinian divergence of $X$ with respect to $\xi$. When there is no risk of confusion, we will write simply $\operatorname{div}_{B}(X)$.

\section{4. (1|1)-supersymmetric sigma model}

Below, we consider a model with target $\mathbb{R}^{1 \mid 1}$, that is, a mapping (scalar superfield) $\sigma:(M, \wedge \mathcal{E}) \rightarrow \mathbb{R}^{1 \mid 1}$, where we consider an odd supermetric on $(M, \wedge \mathcal{E})$ as in Example 2 and the canonical metric of Example 1 on $\mathbb{R}^{1 \mid 1}$.

This imply that $\mathcal{E} \cong \Gamma(T M)$, but this is not a great loss of generality: If $(M, \wedge \mathcal{E})$ admits an odd metric, the existence of the non-degenerate pairing $\kappa: \mathcal{X}(M) \rightarrow \mathcal{E}^{*}$ implies that the dimension of the supermanifold is $(n \mid n)$.

The mapping $\sigma$ can be viewed as a section of the graded submersion

$$
p: \mathbb{R}^{1 \mid 1} \times(M, \wedge \mathcal{E}) \rightarrow(M, \wedge \mathcal{E}),
$$

to which we associate a super-Lagrangian $L \in \mathcal{A}_{J_{G}^{1}(p)}$ (see [13] for the details of this construction) proceeding by analogy with the non-graded case (which is that of harmonic functions). The coordinates in the bundle of superjets $J_{G}^{1}(p)$ will be denoted $\left\{t, \tau, x^{i}, x^{-j}, t_{i}, t_{-j}, \tau_{i}, \tau_{-j}\right\}$. Thus, by taking the pull-back (we write $x^{\alpha}$ collectively for $x^{i}$ and $x^{-j}$, i.e., $\alpha$ runs from $-n, \ldots,-1$ to $1, \ldots, m$ ) of (2) along $\sigma$, we obtain

$$
\begin{aligned}
\sigma^{*} Q & =(-1)^{\alpha \beta} d^{G} x^{\alpha} \otimes d^{G} x^{\beta} \cdot \frac{\partial(t \circ \sigma)}{\partial x^{\alpha}} \frac{\partial(\tau \circ \sigma)}{\partial x^{\beta}} \\
& +(-1)^{(\gamma+1) \delta} d^{G} x^{\gamma} \otimes d^{G} x^{\delta} \cdot \frac{\partial(\tau \circ \sigma)}{\partial x^{\gamma}} \frac{\partial(t \circ \sigma)}{\partial x^{\delta}}
\end{aligned}
$$


As $|-j|=1,|k|=0$, and so on, we could write matricially,

$$
\sigma^{*} Q=\left(\begin{array}{l}
\frac{\partial(t \circ \sigma)}{\partial x^{i}} \frac{\partial(\tau \circ \sigma)}{\partial x^{k}}+\frac{\partial(\tau \circ \sigma)}{\partial x^{2}} \frac{\partial(t \circ \sigma)}{\partial x^{k}} \frac{\partial(t \circ \sigma)}{\partial x^{j}} \frac{\partial(\tau \circ \sigma)}{\partial x^{-1}}-\frac{\partial(\tau \circ \sigma)}{\partial x^{j}} \frac{\partial(t \circ \sigma)}{\partial x^{-l}} \\
\frac{\partial(t \circ \sigma)}{\partial x^{-j}} \frac{\partial(\tau \circ \sigma)}{\partial x^{k}}+\frac{\partial(\tau \circ \sigma)}{\partial x^{-j}} \frac{\partial(t \circ \sigma)}{\partial x^{k}}-\frac{\partial(t \circ \sigma)}{\partial x^{-j}} \frac{\partial(\tau \circ \sigma)}{\partial x^{-l}}+\frac{\partial(\tau \circ \sigma)}{\partial x^{-j}} \frac{\partial(t \circ \sigma)}{\partial x^{-l}}
\end{array}\right) .
$$

Also, from (3) we have $G^{-1}=g^{i j} \cdot \frac{\partial}{\partial x^{i}} \otimes \frac{\partial}{\partial x^{-j}}+g^{k l} \cdot \frac{\partial}{\partial x^{k}} \otimes \frac{\partial}{\partial x^{2}}$, or equivalently, $G^{-1}=\left(\begin{array}{cc}0 & g^{i j} \\ g^{i j} & 0\end{array}\right)$.

We would like to evaluate the action of $G^{-1}$ on $\sigma^{*} Q$ in such a way that the functorial correspondence with the composition of graded morphisms be preserved. To this end, we must use either the action of the metric $G^{-1}$ on $\sigma^{*} Q$ or the notion of matrix product developed in [17]; the result is the following:

$$
\left(\begin{array}{c}
g^{i j}\left(\frac{\partial(t \circ \sigma)}{\partial x^{-j}} \frac{\partial(\tau \circ \sigma)}{\partial x^{k}}+\frac{\partial(\tau \circ \sigma)}{\partial x^{-j}} \frac{\partial(t \circ \sigma)}{\partial x^{k}}\right) g^{i j}\left(-\frac{\partial(t \circ \sigma)}{\partial x^{-j}} \frac{\partial(\tau \circ \sigma)}{\partial x^{-l}}+\frac{\partial(\tau \circ \sigma)}{\partial x^{-j}} \frac{\partial(t \circ \sigma)}{\partial x^{-l}}\right) \\
g^{i j}\left(\frac{\partial(t \circ \sigma)}{\partial x^{i}} \frac{\partial(\tau \circ \sigma)}{\partial x^{k}}+\frac{\partial(\tau \circ \sigma)}{\partial x^{i}} \frac{\partial(t \circ \sigma)}{\partial x^{k}}\right) g^{i j}\left(\frac{\partial(t \circ \sigma)}{\partial x^{j}} \frac{\partial(\tau \circ \sigma)}{\partial x^{-l}}-\frac{\partial(\tau \circ \sigma)}{\partial x^{j}} \frac{\partial(t \circ \sigma)}{\partial x^{-l}}\right)
\end{array}\right) .
$$

If we were to take the supertrace of this supermatrix, we would obtain 0 , as the following computation shows:

$$
\begin{aligned}
\operatorname{Str}\left(G^{-1} \cdot \sigma^{*} Q\right) & =\operatorname{Tr}\left(g^{i j}\left(\frac{\partial(t \circ \sigma)}{\partial x^{-j}} \frac{\partial(\tau \circ \sigma)}{\partial x^{k}}+\frac{\partial(\tau \circ \sigma)}{\partial x^{-j}} \frac{\partial(t \circ \sigma)}{\partial x^{k}}\right)\right) \\
& -\operatorname{Tr}\left(g^{i j}\left(\frac{\partial(t \circ \sigma)}{\partial x^{j}} \frac{\partial(\tau \circ \sigma)}{\partial x^{-l}}-\frac{\partial(\tau \circ \sigma)}{\partial x^{j}} \frac{\partial(t \circ \sigma)}{\partial x^{-l}}\right)\right) \\
& =g^{i j}\left(\frac{\partial(t \circ \sigma)}{\partial x^{-j}} \frac{\partial(\tau \circ \sigma)}{\partial x^{i}}+\frac{\partial(\tau \circ \sigma)}{\partial x^{-j}} \frac{\partial(t \circ \sigma)}{\partial x^{i}}\right) \\
& -g^{k l}\left(\frac{\partial(t \circ \sigma)}{\partial x^{l}} \frac{\partial(\tau \circ \sigma)}{\partial x^{-k}}-\frac{\partial(\tau \circ \sigma)}{\partial x^{l}} \frac{\partial(t \circ \sigma)}{\partial x^{-k}}\right) \\
& =g^{i j} \frac{\partial(t \circ \sigma)}{\partial x^{-j}} \frac{\partial(\tau \circ \sigma)}{\partial x^{i}}+g^{k l} \frac{\partial(\tau \circ \sigma)}{\partial x^{l}} \frac{\partial(t \circ \sigma)}{\partial x^{-k}} \\
& +g^{i j} \frac{\partial(\tau \circ \sigma)}{\partial x^{-j}} \frac{\partial(t \circ \sigma)}{\partial x^{i}}-g^{k l} \frac{\partial(t \circ \sigma)}{\partial x^{l}} \frac{\partial(\tau \circ \sigma)}{\partial x^{-k}} \\
& =g^{i j} \frac{\partial(t \circ \sigma)}{\partial x^{-j}} \frac{\partial(\tau \circ \sigma)}{\partial x^{i}}-g^{k l} \frac{\partial(t \circ \sigma)}{\partial x^{-k}} \frac{\partial(\tau \circ \sigma)}{\partial x^{l}} \\
& +g^{i j} \frac{\partial(\tau \circ \sigma)}{\partial x^{-j}} \frac{\partial(t \circ \sigma)}{\partial x^{i}}-g^{k l} \frac{\partial(\tau \circ \sigma)}{\partial x^{-k}} \frac{\partial(t \circ \sigma)}{\partial x^{l}}=0 .
\end{aligned}
$$

Due to this, instead of Str we will take the usual contraction of $(1,1)$ -

${ }^{\text {a } U p p e r ~ l e f t ~ c o r n e r ~ c o r r e s p o n d s ~ t o ~ t h e ~ f a c t o r ~ o f ~} d^{G} x^{i} \otimes d^{G} x^{k}$, upper right to $d^{G} x^{j} \otimes d^{G} x^{-l}$, lower left to $d^{G} x^{-j} \otimes d^{G} x^{k}$, and lower right to $d^{G} x^{-j} \otimes d^{G} x^{-l}$. 
supertensors, which amounts to

$$
\begin{aligned}
C_{1}^{1}\left(G^{-1} \cdot \sigma^{*} Q\right) & =\operatorname{Tr}\left(g^{i j}\left(\frac{\partial(t \circ \sigma)}{\partial x^{-j}} \frac{\partial(\tau \circ \sigma)}{\partial x^{k}}+\frac{\partial(\tau \circ \sigma)}{\partial x^{-j}} \frac{\partial(t \circ \sigma)}{\partial x^{k}}\right)\right) \\
& +\operatorname{Tr}\left(g^{i j}\left(\frac{\partial(t \circ \sigma)}{\partial x^{j}} \frac{\partial(\tau \circ \sigma)}{\partial x^{-l}}-\frac{\partial(\tau \circ \sigma)}{\partial x^{j}} \frac{\partial(t \circ \sigma)}{\partial x^{-l}}\right)\right) \\
& =g^{i j}\left(\frac{\partial(t \circ \sigma)}{\partial x^{-j}} \frac{\partial(\tau \circ \sigma)}{\partial x^{i}}+\frac{\partial(\tau \circ \sigma)}{\partial x^{-j}} \frac{\partial(t \circ \sigma)}{\partial x^{i}}\right) \\
& +g^{k l}\left(\frac{\partial(t \circ \sigma)}{\partial x^{l}} \frac{\partial(\tau \circ \sigma)}{\partial x^{-k}}-\frac{\partial(\tau \circ \sigma)}{\partial x^{l}} \frac{\partial(t \circ \sigma)}{\partial x^{-k}}\right) \\
& =g^{i j} \frac{\partial(t \circ \sigma)}{\partial x^{-j}} \frac{\partial(\tau \circ \sigma)}{\partial x^{i}}-g^{k l} \frac{\partial(\tau \circ \sigma)}{\partial x^{l}} \frac{\partial(t \circ \sigma)}{\partial x^{-k}} \\
& +g^{i j} \frac{\partial(\tau \circ \sigma)}{\partial x^{-j}} \frac{\partial(t \circ \sigma)}{\partial x^{i}}+g^{k l} \frac{\partial(t \circ \sigma)}{\partial x^{l}} \frac{\partial(\tau \circ \sigma)}{\partial x^{-k}} \\
& =2 g^{i j} \frac{\partial(t \circ \sigma)}{\partial x^{-j}} \frac{\partial(\tau \circ \sigma)}{\partial x^{i}}+2 g^{i j} \frac{\partial(t \circ \sigma)}{\partial x^{i}} \frac{\partial(\tau \circ \sigma)}{\partial x^{-j}} .
\end{aligned}
$$

That is, we have arrived at the result

$$
\frac{1}{2} C_{1}^{1}\left(G^{-1} \cdot \sigma^{*} Q\right)=g^{i j}\left(\frac{\partial(t \circ \sigma)}{\partial x^{-j}} \frac{\partial(\tau \circ \sigma)}{\partial x^{i}}+\frac{\partial(t \circ \sigma)}{\partial x^{i}} \frac{\partial(\tau \circ \sigma)}{\partial x^{-j}}\right),
$$

and this suggests to take the following superlagrangian $L \in \mathcal{A}_{J_{G}^{1}(p)}$ to study the supersymmetric sigma model (where we introduce the obvious notations $t_{\alpha}=\frac{\partial(t \circ \sigma)}{\partial x^{\alpha}}$, $\left.\tau_{\beta}=\frac{\partial(\tau \circ \sigma)}{\partial x^{\beta}}\right)$ :

$$
L=g^{i j}\left(t_{-j} \tau_{i}+t_{i} \tau_{-j}\right)
$$

Remark 3. The superlagrangian $L$ is homogeneous of even degree. In Physics literature, it is common to write $t \circ \sigma=\phi$ and $\tau \circ \sigma=\psi$, so equation (11) would be

$$
\frac{1}{2} C_{1}^{1}\left(G^{-1} \cdot \sigma^{*} Q\right)=g^{i j}\left(\frac{\partial \phi}{\partial x^{-j}} \frac{\partial \psi}{\partial x^{i}}+\frac{\partial \phi}{\partial x^{i}} \frac{\partial \psi}{\partial x^{-j}}\right)
$$

and the Lagrangian density

$$
L=g^{i j}\left(\phi_{-j} \psi_{i}+\phi_{i} \psi_{-j}\right)
$$

\section{The associated variational problem and harmonic superfunctions}

Next, we want to study the Euler-Lagrange equations corresponding to the Berezinian problem defined by $L$. To this end, we recall that $G$ determines a global section $\xi_{G}$ of the Berezinian sheaf $\operatorname{Ber}(\mathcal{A})=\operatorname{Ber}(\wedge \mathcal{E})$, which is called the Riemannian Berezinian volume element associated to $G$. In coordinates:

$$
\xi_{G}=\left[d^{G} x^{1} \wedge \cdots \wedge d^{G} x^{m} \otimes \frac{\partial}{\partial x^{-1}} \circ \cdots \circ \frac{\partial}{\partial x^{-n}}\right]|G|
$$


where $|G|=\sqrt{\operatorname{Ber}\left(G_{\alpha \beta}\right)}$ and $\operatorname{Ber}$ is the Berezinian determinant (note that $|G|$ is even and it only depends on the even coordinates $x^{i}$ ). The Euler-Lagrange equations are

$$
\begin{aligned}
& \frac{\partial \lambda}{\partial t}-\frac{d}{d x^{i}} \frac{\partial \lambda}{\partial t_{i}}-\frac{d}{d x^{-j}} \frac{\partial \lambda}{\partial t_{-j}}=0,1 \leq i \leq m, 1 \leq j \leq n \\
& \frac{\partial \lambda}{\partial \tau}-\frac{d}{d x^{i}} \frac{\partial \lambda}{\partial \tau_{i}}+\frac{d}{d x^{-j}} \frac{\partial \lambda}{\partial \tau_{-j}}=0,1 \leq i \leq m, 1 \leq j \leq n,
\end{aligned}
$$

with $\lambda=|G| \cdot L$.

Taking the explicit expression (2) or (3) into account, it is readily seen that these equations reduce respectively to

$$
\begin{aligned}
\frac{1}{|G|} \frac{\partial|G|}{\partial x^{k}} g^{k j} \tau_{-j}+\frac{\partial g^{k j}}{\partial x^{k}} \tau_{-j}+g^{k j} \tau_{k,-j}+g^{k j} \tau_{-k j} & =0 \\
\frac{1}{|G|} \frac{\partial|G|}{\partial x^{k}} g^{k j} t_{-j}+\frac{\partial g^{k j}}{\partial x^{k}} t_{-j}+g^{k j} t_{k,-j}+g^{k j} & =0 .
\end{aligned}
$$

Classically, the Euler-Lagrange variational equations for the sigma model with target manifold $\mathbb{R}$ are those of the harmonic functions, characterized by $\Delta f=0$, where $\Delta$ is the ordinary Laplacian: $\Delta f=\operatorname{div}(\operatorname{grad} f)($ see [16[15]). We would like to show that this is still true in the graded setting, that is, that equations (10) when evaluated on sections are the local expression of the harmonic superfunctions, aside from constant factors. Of course, to define the super-Laplacian $\Delta$ one must specify first what is meant by div and grad in a supermanifold.

If $f \in \wedge \mathcal{E}$ is a superfunction on $(M, \wedge \mathcal{E})$ with graded metric $G$, its gradient is defined as the supervector field given by $\langle\operatorname{grad} f, D\rangle=D(f)$, for all $D \in \operatorname{Der}(\wedge \mathcal{E})$.

Proposition 4. For a superfunction $f \in \wedge \mathcal{E}$, the following local expression holds true,

$$
\operatorname{grad} f=g^{i j} \frac{\partial f}{\partial x^{-j}} \frac{\partial}{\partial x^{i}}+g^{k l} \frac{\partial f}{\partial x^{k}} \frac{\partial}{\partial x^{-l}} .
$$

Proof. Indeed, making use of the $\wedge \mathcal{E}$-bilinearity of $G$ and the explicit form (3), if $\operatorname{grad} f=A^{\alpha} \frac{\partial}{\partial x^{\alpha}}$, then

$$
\frac{\partial f}{\partial x^{-j}}=\left\langle\operatorname{grad} f, \frac{\partial}{\partial x^{-j}}\right\rangle=A^{\alpha}\left\langle\frac{\partial}{\partial x^{\alpha}}, \frac{\partial}{\partial x^{-j}}\right\rangle=A^{i} g_{i j} .
$$

Hence $A^{i}=g^{i j} \frac{\partial f}{\partial x^{-j}}$, and similarly for $A^{-l}=g^{k l} \frac{\partial f}{\partial x^{k}}$.

Next, we study the divergence. If we consider the Riemannian Berezinian $\xi_{G}=$ $\xi|G|$ and compute the divergence of $D \in \operatorname{Der}(\wedge \mathcal{E})$ with respect to it, we obtain the following (recall the properties of the Lie derivative of sections of the Berezinian sheaf from section 3):

Proposition 5. For any $D \in \operatorname{Der}(\wedge \mathcal{E})$, we have

$$
\operatorname{div} D=(-1)^{|D||\xi|} \frac{1}{|G|} \frac{\partial}{\partial x^{\alpha}}\left(|G| \cdot D^{\alpha}\right) .
$$


Proof. It goes as follows:

$$
\begin{aligned}
\mathcal{L}_{D}^{G} \xi_{G} & =\mathcal{L}_{D^{\alpha} \frac{\partial}{\partial x^{\alpha}}}^{G}(\xi \cdot|G|) \\
& =(-1)^{\alpha|\xi|} \mathcal{L}_{\frac{\partial}{\partial x^{\alpha}}}^{G}\left(\xi \cdot|G| \cdot D^{\alpha}\right) \\
& =(-1)^{\alpha|\xi|}\left(\mathcal{L}_{\frac{\partial}{\partial x^{\alpha}}}^{G}(\xi) \cdot\left(|G| \cdot D^{\alpha}\right)+(-1)^{\alpha|\xi|} \xi \cdot \frac{\partial}{\partial x^{\alpha}}\left(|G| \cdot D^{\alpha}\right)\right) \\
& =\xi \cdot \frac{\partial}{\partial x^{\alpha}}\left(|G| \cdot D^{\alpha}\right) \\
& =\xi \cdot|G| \cdot \frac{1}{|G|} \frac{\partial}{\partial x^{\alpha}}\left(|G| \cdot D^{\alpha}\right) \\
& =\xi_{G} \cdot \frac{1}{|G|} \frac{\partial}{\partial x^{\alpha}}\left(|G| \cdot D^{\alpha}\right) .
\end{aligned}
$$

Finally, from (2) and (3), we obtain the expression for the super-Laplacian of a superfunction $f \in \wedge \mathcal{E}$, namely,

Proposition 6. For any superfunction $f \in \wedge \mathcal{E}$, the equation $\Delta(f)=0$ is equivalent to:

$$
\frac{1}{|G|} \frac{\partial|G|}{\partial x^{i}} g^{i j} f_{-j}+\frac{\partial g^{i j}}{\partial x^{i}} f_{-j}+g^{i j} f_{i,-j}+g^{i j} f_{-i, j}=0 .
$$

Proof. By a direct computation:

$$
\begin{aligned}
(-1)^{(|f|+1)|\xi|} \Delta(f) & =(-1)^{(|f|+1)|\xi|} \operatorname{div}(\operatorname{grad} f) \\
& =\frac{1}{|G|} \frac{\partial}{\partial x^{\alpha}}\left(|G| \cdot(\operatorname{grad} f)^{\alpha}\right) \\
& =\frac{1}{|G|}\left(\frac{\partial}{\partial x^{i}}\left(|G| \cdot g^{i j} \frac{\partial f}{\partial x^{-j}}\right)+\frac{\partial}{\partial x^{-i}}\left(|G| \cdot g^{i k} \frac{\partial f}{\partial x^{k}}\right)\right) \\
& =\frac{1}{|G|} \frac{\partial|G|}{\partial x^{i}} g^{i j} f_{-j}+\frac{\partial g^{i j}}{\partial x^{i}} f_{-j}+g^{i j} f_{i,-j}+g^{i j} f_{-i, j} .
\end{aligned}
$$

Separating the even and odd factors, we obtain (1) precisely when evaluated on sections of $p: \mathbb{R}^{1 \mid 1} \times(M, \wedge \mathcal{E}) \rightarrow(M, \wedge \mathcal{E})$. We have thus achieved the following characterization:

Theorem 7. The harmonic superfunctions are precisely the solutions to the EulerLagrange equations of the (1|1)-supersymmetric sigma model.

\section{Acknowledgments}

Partially supported by the Ministerio de Educación y Ciencia of Spain, under grants MTM2008 - 01386 and MTM2005 - 04947. JAV also supported by a FAI-UASLP grant C07-FAI-04-18.20 and a SEP-CONACyT project CB (J2) 2007-1 code 78791. 


\section{References}

[1] S. Aoyama, Quantization of the topological $\sigma$-model and the master equation of the $B V$ formalism. Modern Phys. Lett. A9 (1994) no. 6, $491-500$.

[2] I. A. Batalin, K. Bering, Odd scalar curvature in anti-Poisson geometry. arXiv hepth:0712.3699v2 (2 January 2008).

[3] P. Deligne et al. (Eds.), Quantum Fields and Strings: A Course For Mathematicians, 2 vols., American Mathematical Society, Providence, 1999.

[4] J. Eells, L. Lemaire, A report on harmonic maps. Bull. London Math. Soc. 10 (1978) 1.

[5] J. Eells, J. H. Sampson, Harmonic mappings of Riemannian manifolds. Amer. J. Math. 86 (1964), 109.

[6] H. Khudaverdian, T. Voronov, On odd Laplace operators. Lett. Math. Phys. 62 (2002) no. $2,127-142$.

[7] H. Khudaverdian, T. Voronov, On odd Laplace operators II. Geometry, Topology and Mathematical Physics, 179 - 205. Amer. Math. Soc. Transl. Ser. 2 212. Amer. Math. Soc., Providence (RI) 2004.

[8] H. Khudaverdian, T. Voronov, Geometry of differential operators, odd Laplacians, and homotopy algebras. J. Nonlinear Math. Phys. 11 (2004) suppl., 217 - 227.

[9] Y. Kosmann-Schwarzbach, J. Monterde, Divergence operators and odd Poisson brackets. Ann. Inst. Fourier 52 (2002) no. 2, 419 - 456.

[10] J. Monterde and O. A. Sánchez-Valenzuela, The exterior derivative as a Killing vector field, Israel J. of Math. 93 (1996), $157-170$.

[11] J. Monterde, O. A. Sánchez-Valenzuela, Graded metrics adapted to splittings. Israel J. Math. 99 (1997), $231--270$.

[12] J. Monterde, J. A. Vallejo, The symplectic structure of Euler-Lagrange superequations and Batalin-Vilkoviski formalism. J. Phys. A: Math. and General A36 (2003) 4993 5009.

[13] J. Monterde, J. Muñoz-Masqué, J. A. Vallejo, The Poincaré-Cartan form in superfield theory. Int. J. Geom. Methods Mod. Phys. 3 (2006) no. 4, $775-822$.

[14] J. Muñoz-Masqué, J.A. Vallejo, Harmonicity in supermanifolds and sigma models. To appear in Proceedings of the XVII Fall Workshop on Geometry and Physics.

[15] S. Nishikawa, Variational problems in geometry. Translations of mathematical monographs 205 American Mathematical Society. Providence, RI (2002).

[16] J. Rawnsley, Noether's theorem for harmonic maps. In Differential geometric methods in mathematical physics. S. Sternberg, editor. D. Reidel, Dordrecht (1984).

[17] O. A. Sánchez-Valenzuela, Matrix computations in linear superalgebra. Linear Algebra Appl. 111 (1988), 151 - 181.

[18] G. Sardanashvily, Supermetrics on supermanifolds. Int. J. Geom. Methods Mod. Phys. v5 (2008) $271-286$.

[19] A. Schwarz, Geometry of Batalin-Vilkovisky quantization. Comm. Math. Phys. 155 (1993) no. 2, $249-260$.

[20] E. Witten, A note on the antibracket formalism. Mod. Phys. Lett. A 5 no. 7 (1990) $487-494$. 